\title{
THE EFFECTS OF COMPUTER BASED COGNITIVE REHABILITATION IN PATIENTS WITH SYMPTOMS OF VISUOSPATIAL NEGLECT OR HEMIANOPSIA AFTER STROKE: A RANDOMIZED, CONTROLLED, UNBLINDED CROSS-OVER PILOT-STUDY.
}

\section{BACKGROUND}

Visuospatial neglect and hemianopsias are symptoms occuring after certain types of stroke, that have a negative impact on visuospatial functions. As a result, both symptoms have a negative impact on quality of life and ADL-functions, and are predictors of long-term disability. No rehabilitation methods can yet be recommended over others for visuospatial neglect or hemianopsias beyond scientific doubt.

\section{PURPOSE OF THIS TRIAL}

The purpose of this pilot trial was to investigate the effects of a computer based cognitive rehabilitation (CBCR) method in patients with visuospatial neglect or homonymous hemianopsia in the subacute phase following stroke.

\section{METHOD}

\section{Description of $C B C R$-intervention:}

CBCR was delivered by a commercially available program: 'Scientific Braintraining PRO' [1]. Among other tasks, the program consists of five different computerized training tasks all designed to train visual scanning, visual attention and mental rotation. An example of a visual scanning task is provided in figure 1. All Patients were instructed to train 45 minutes every second day for three weeks as a supplement to their guideline rehabilitation

\begin{tabular}{l|l|l|l|l|l|l}
\hline ID & Sex & Group & Age at scan & Earlier lesions & Acute/ Subacute lesion & Symptom \\
\hline $\mathbf{1}$ & Female & Early & 53 & Several bilateral & Right, posterior & Left neglect \\
\hline $\mathbf{2}$ & Male & Early & 63 & No & Right, posterior & Left hemianopsia \\
\hline $\mathbf{3}$ & Female & Early & 71 & No & Right, medial & Left neglect \\
\hline $\mathbf{5}$ & Male & Early & 49 & No & Left, posterior & $\begin{array}{l}\text { Right } \\
\text { hemianopsia }\end{array}$ \\
\hline $\mathbf{6}$ & Female & Early & 69 & Several bilateral & Several Bilateral & Right neglect \\
\hline $\mathbf{7}$ & Male & Late & $\mathbf{7 4}$ & Several bilateral & Left, posterior & Left neglect \\
\hline $\mathbf{8}$ & Female & Late & 81 & Right, medial & Right, medial & Left neglect \\
\hline $\mathbf{9}$ & Male & Late & $\mathbf{7 3}$ & No & Right, medial & Left neglect \\
\hline $\mathbf{1 0}$ & Female & Late & 66 & Left medial & Right, posterior & Left hemianopsia \\
\hline $\mathbf{1 3}$ & Male & Early & 71 & Left anterior & Left, medial & Right neglect \\
\hline $\mathbf{1 5}$ & Male & Late & 62 & No & Right, medial & Left neglect \\
\hline $\mathbf{1 6}$ & Male & Late & $\mathbf{7 8}$ & No & Several & Right neglect \\
\hline $\mathbf{1 7}$ & Female & Late & 51 & No & Right, medial & Left neglect \\
\hline $\mathbf{1 8}$ & Female & Early & 40 & No & Right, medial & Left neglect \\
\hline
\end{tabular}

Figure 2: Patient characteristics. 4 patients were excluded because they did not train during the intervention-period or because they withdraw their consent.

Participants:

Fourteen patients were randomly assigned to an early or late CBCR intervention targeting visuospatial symptoms in a cross-over design. All patients were included within 40 days of stroke onset. Patient characteristics are provided in figure 2. Patients were included if a doctor or neuropsychologist described visuospatial neglect or hemianopsia in the journal after stroke onset, if the patient did not have a

neurodegenerative disease or other comorbidities at risk of influencing the patient's ability to profit from $C B C R$, if the patient was judged both motorically and cognitively capable of CBCR and was able to give informed consent both verbally and in writing.

\section{Course of the trial for each participant:}

The course of the trial was six weeks for each patient. All patients underwent baseline assessment at inclusion with a neuropsychological test-battery specifically designed for this trial (See below). The early intervention group (El group) received CBCR starting immediately after baseline-assessment for three weeks ( $\mathrm{m}=19$ days after stroke onset), and the late intervention group (LI group) started a three-week CBCR intervention three weeks after baseline-assessment $(m=44$ days after stroke onset). All patients were re-assessed with the same neuropsychological test-battery at three weeks and six weeks.

\section{Neuropsychological assessment:}

The primary outcome of this trial was a computerized version similar to the Star-cancellation test, which is a common paper-and-pencil test of visuospatial neglect and hemianopisia. The computerized version is called 'butterfly finding' and is found in the neuropsychological assessment app CABpad [2]

The assessment-battery also included:

The block design task from WAIS-IV (Visuoconstructive abilities not requiring grafomotoric abilities)

The MSU drawing task (Visuoconstructive abilities requiring grafomotoric abilities)

The street completion test (Visual synthesis)

Scl-90-r (Mental health questionnaire)

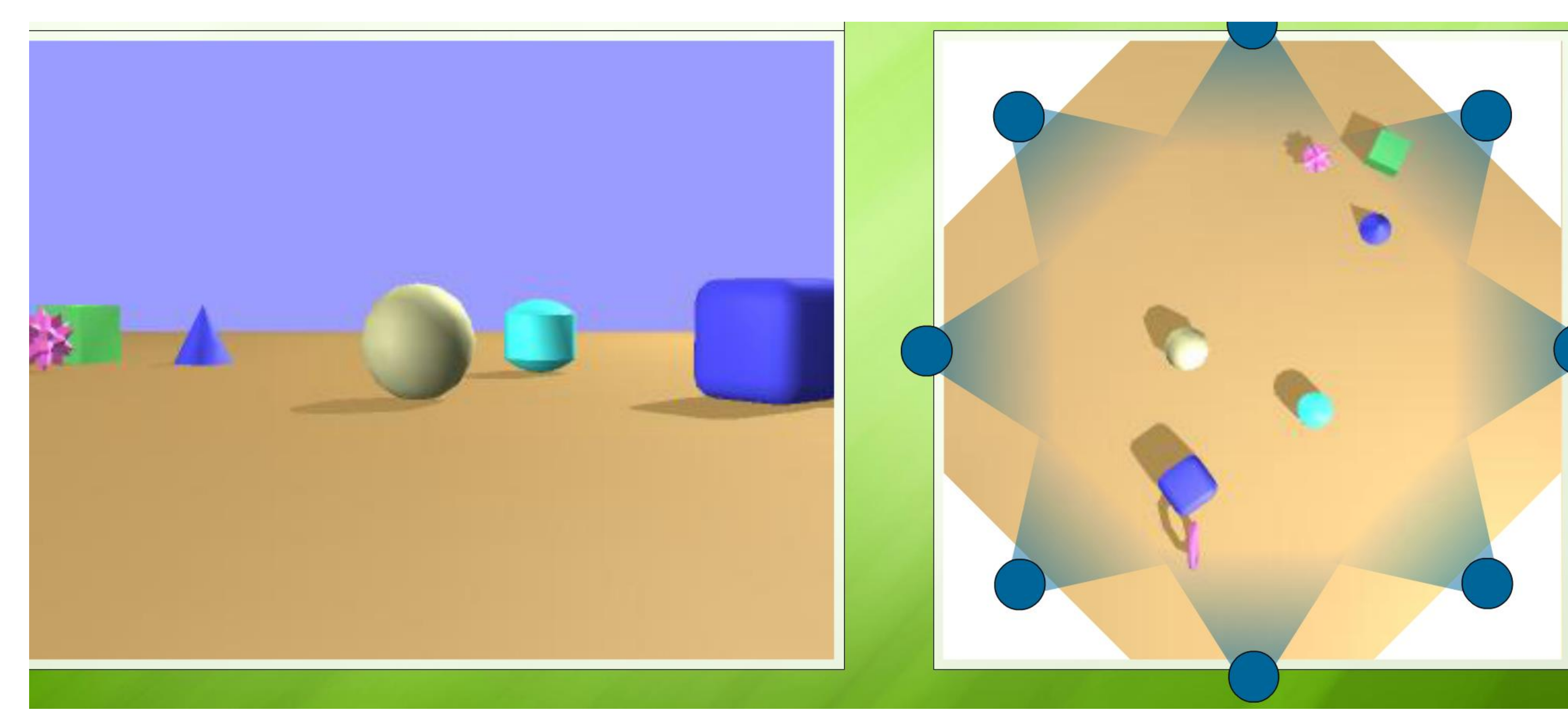

Figure 1: An example of one of the five training-tasks of visuospatial training. The picture from the presented visual field. Every blue circle constitutes a possible position.

\section{RESULTS}

Groups were balanced on baseline characteristics. The El group showed a significant reduction in neglect score on the primary outcome test 'Butterfly finding on CABpad' between baseline and after three weeks of training $\left(p=0.018^{*}, r=0.89\right)$, with no significant difference after three weeks of pause from CBCR at week six $(p=0.735, r=0.13$ I although there was a trend in a negative direction. The neglect score did not change significantly in the LI group, neither during the waiting list period during week one to three $(p=0.237, r=0.44)$ nor during training during week three to six $(p=0.116, r=0.59)$, though an insignificant trend in this direction was observed both at week three and week six. The difference between the development of the El group and $\mathrm{LI}$ group during the first three weeks was not significant $(p=0.383, r=0.26)$, which might be due to a lack of statistical power in this trial. The difference in improvement during training periods was not significant between the $\mathrm{El}$ and $\mathrm{LI}$ group $(p=0.259, r=0.32)$

\section{CONCLUSIONS}

CBCR improved visuospatial symptoms after stroke significantly when administered early in the subacute phase after stroke. The same significant effect was not found when CBCR was administered later in the rehabilitation. However, the difference in the development of the $\mathrm{El}$ group and LI group during the first three weeks was not significant, which could be due to a lack of statistical power. The study was small and confirmation is needed.

\section{LIMITATIONS AND FUTURE DIRECTIONS}

This pilot-study was small and under-powered and the outcome assessor was not blind to the group allocation of patients. Furtheremore, the study assessed outcome with a neuropsychological test-battery, and more ecological measures of ADL-functions are lacking. Further research is needed to confirm the findings of this pilot-study and clarify to what extend CBCR is an effective intervention in the rehabilitation method for visuospatial neglect and hemianopsia.

\section{REFERENCES}

1. www.happy-neuron.com

2. Willer, L., Pedersen, P. M., Forchhammer, H. B \& Christensen, H. (2016). Cognitive assessment at bedside for iPad: A preliminary validation of a nove cognitive test for stroke patients. European Stroke Journal, 1(4), 294-301.

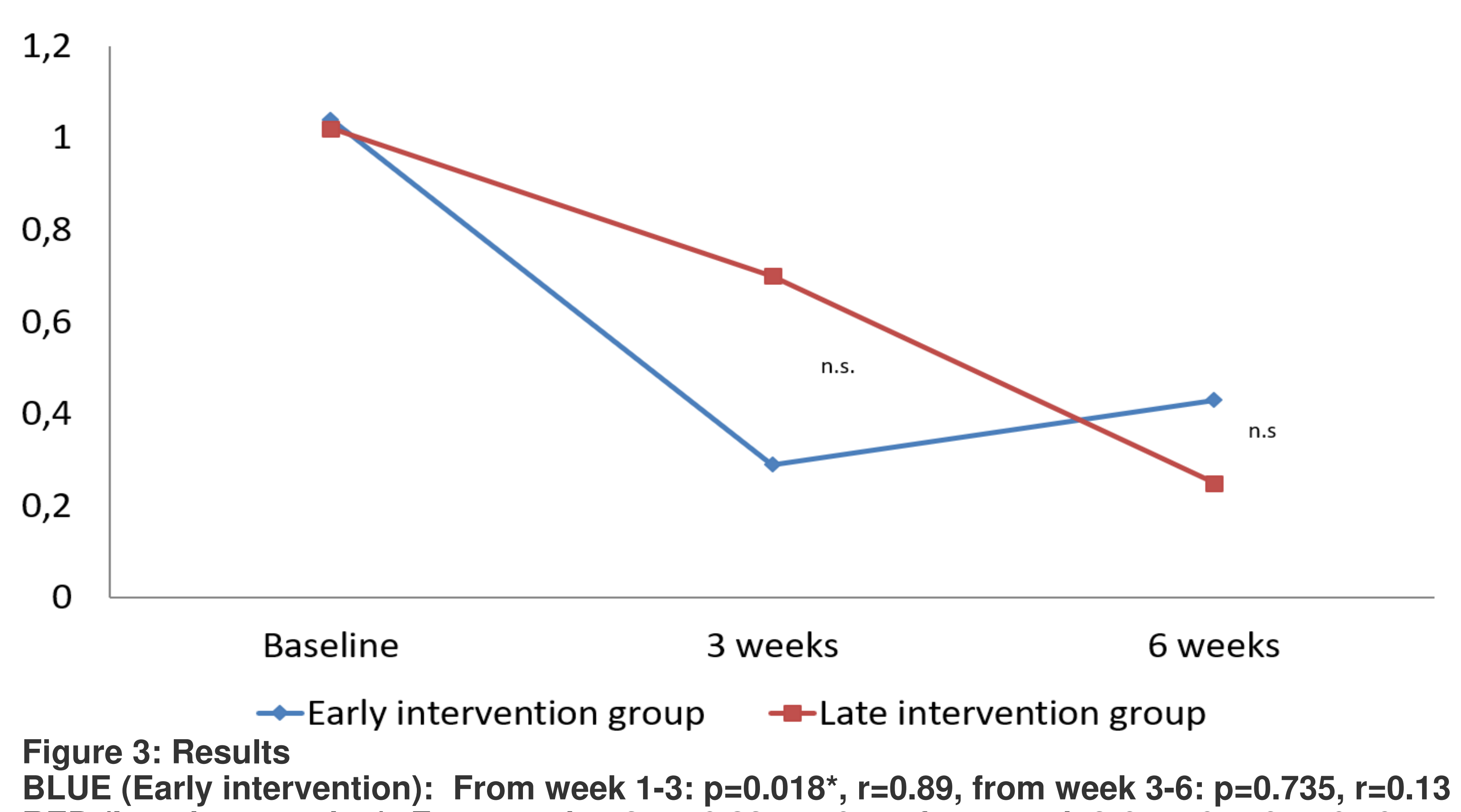

